\title{
Diversity in mycotoxin biodegradation vs. detoxification within Actinobacteria: create no greater evil
}

\section{Laura De Mets' ${ }^{1}$ Kris Audenaert², Leen De Gelder ${ }^{1}$}

${ }^{1}$ Lab. of Environmental Biotechnology, Dept. of Biotechnology, Faculty of Bioscience Engineering, Ghent Univeristy,

2 Laboratory of Applied Mycology and Phenomics, Dept. of Crop Protection, Faculty of Bioscience Engineering, Ghent University

\section{Introduction}

Fungal contamination of crops cannot only lead to lowered nutrional value, but also poses a serious health treath as the fungi can produce mycotoxins. This wide range of secondary metabolites can cause health problems ranging from vomiting to cancer and even death. Next to prevention of fungal contamination, and lowering the disease pressure once the fungus is already present, a huge effort is made to find solutions to remediate contaminated batches.

In this research, we focus on the non-steroidal estrogenic mycotoxin zearalenone. This toxin, harbouring a macrocyclic lactone ring and a benzene moiety, can bind to the estrogenic receptor, thus causing hyperestrogenic issues such as infertility.

As Actinobacteria are known for the degradation of recalcitrant pollutants such as polyaromatic hydrocarbons, they offer great potential for bioremediation of zearalenone. Here, we present a screening of fifty-three Actinobacteria for the degradation and detoxification of zearalenone.

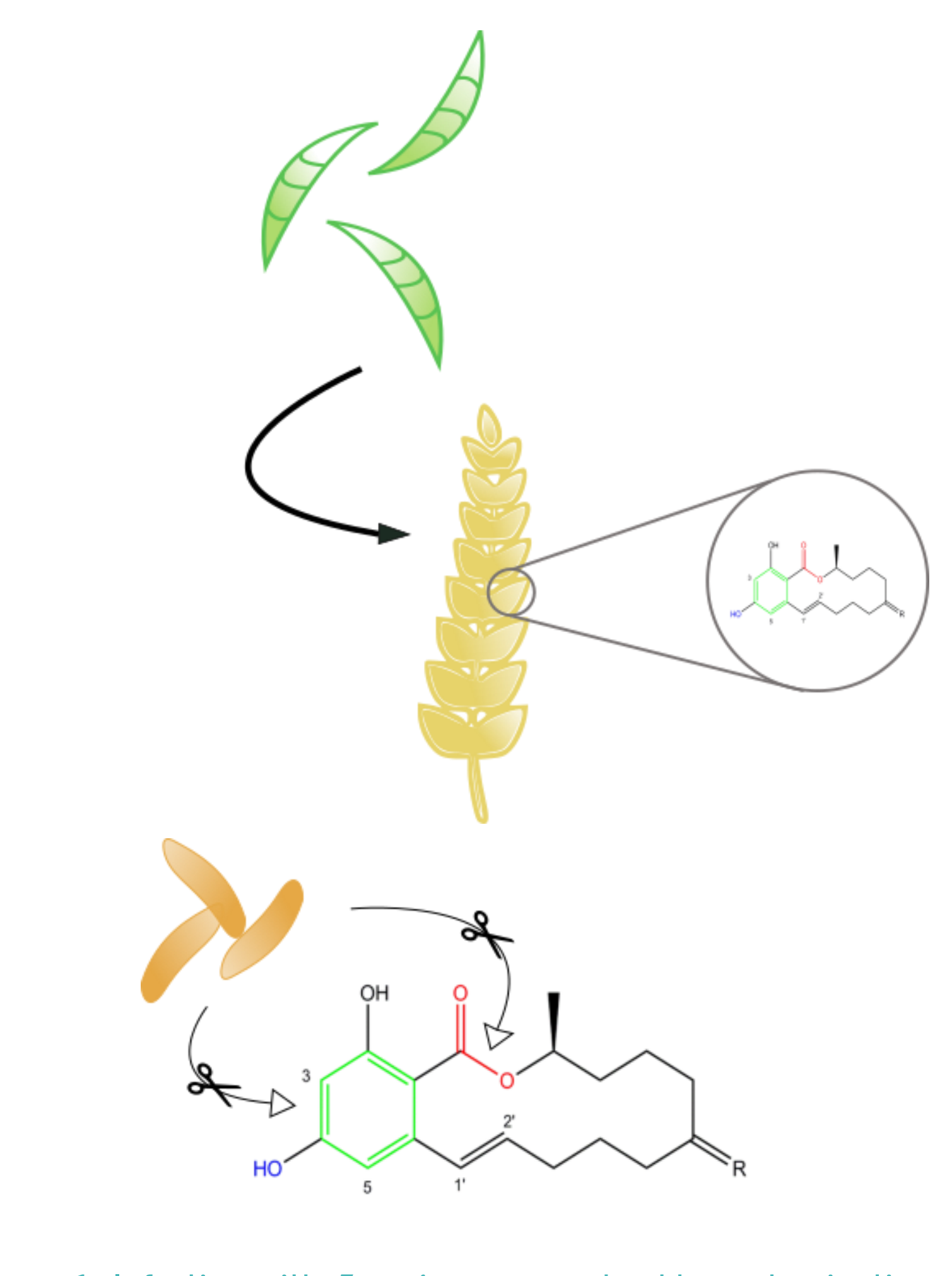

igure 1: Infection With fusarium spp. can lead to contamming tion

\section{Actinobacteria: Catabolic masters}

Actinobacteria have been shown to degrade a wide variety of recalcitrant polluants.

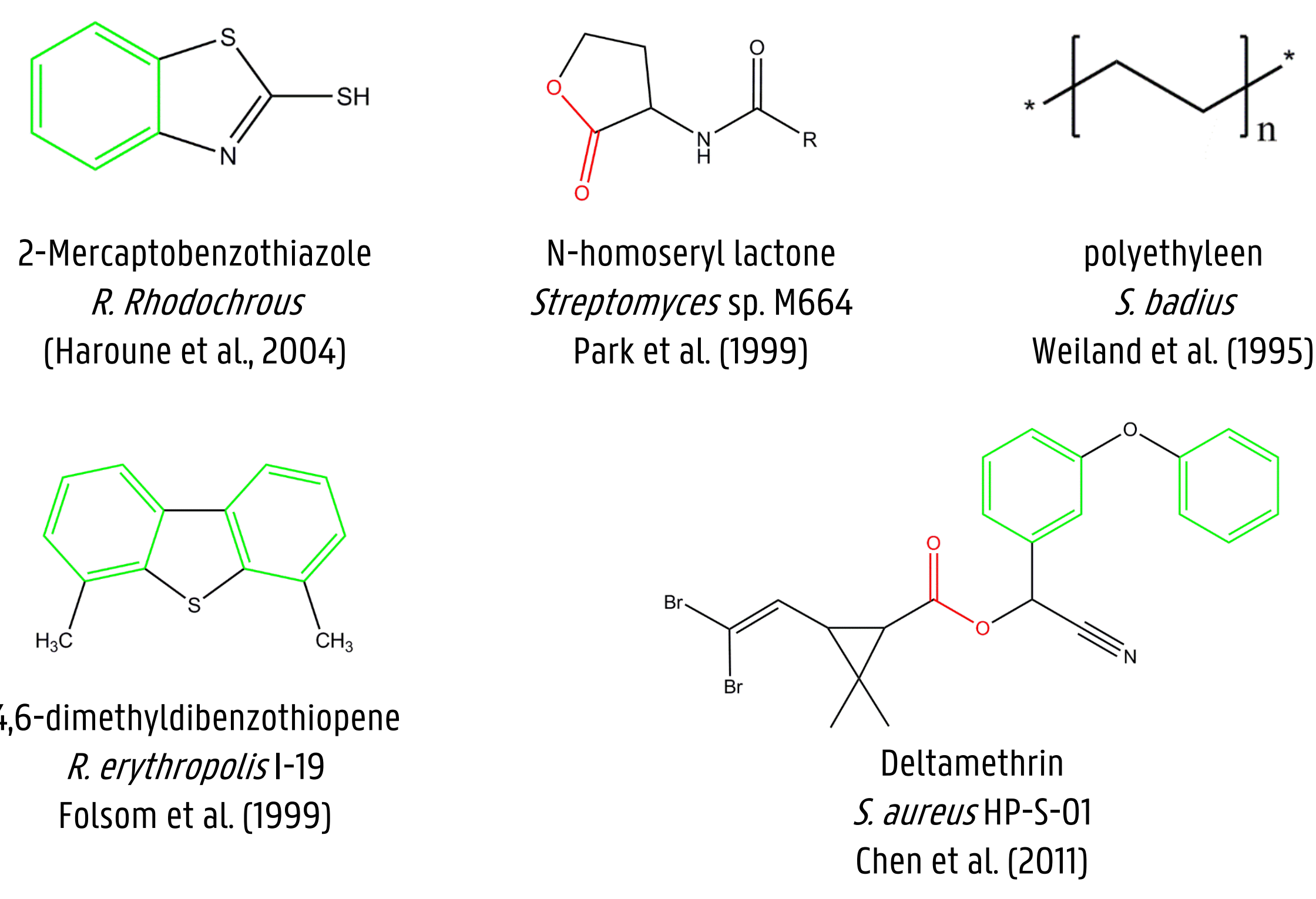

\section{Screening workflow}

Dereplication of strain collection by protein profiling with MALDI-TOF

Out of 81 analysed strains, 53 were selected for screening in a degradation assay.

\section{$5 \mathrm{mg} / \mathrm{LZEN}$ in LB broth}

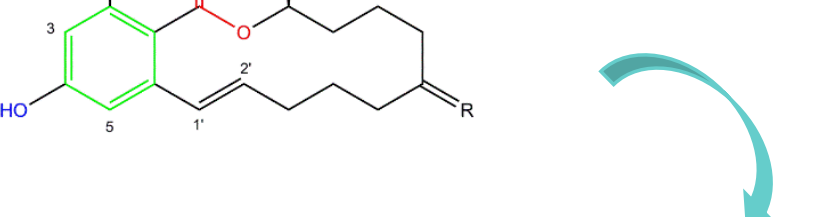

Inoculant strain
Degradation?

\section{Detoxification?}

\section{Results}

Degradation of ZEN can lead to either a decrease or an increase in estrogenic toxicity

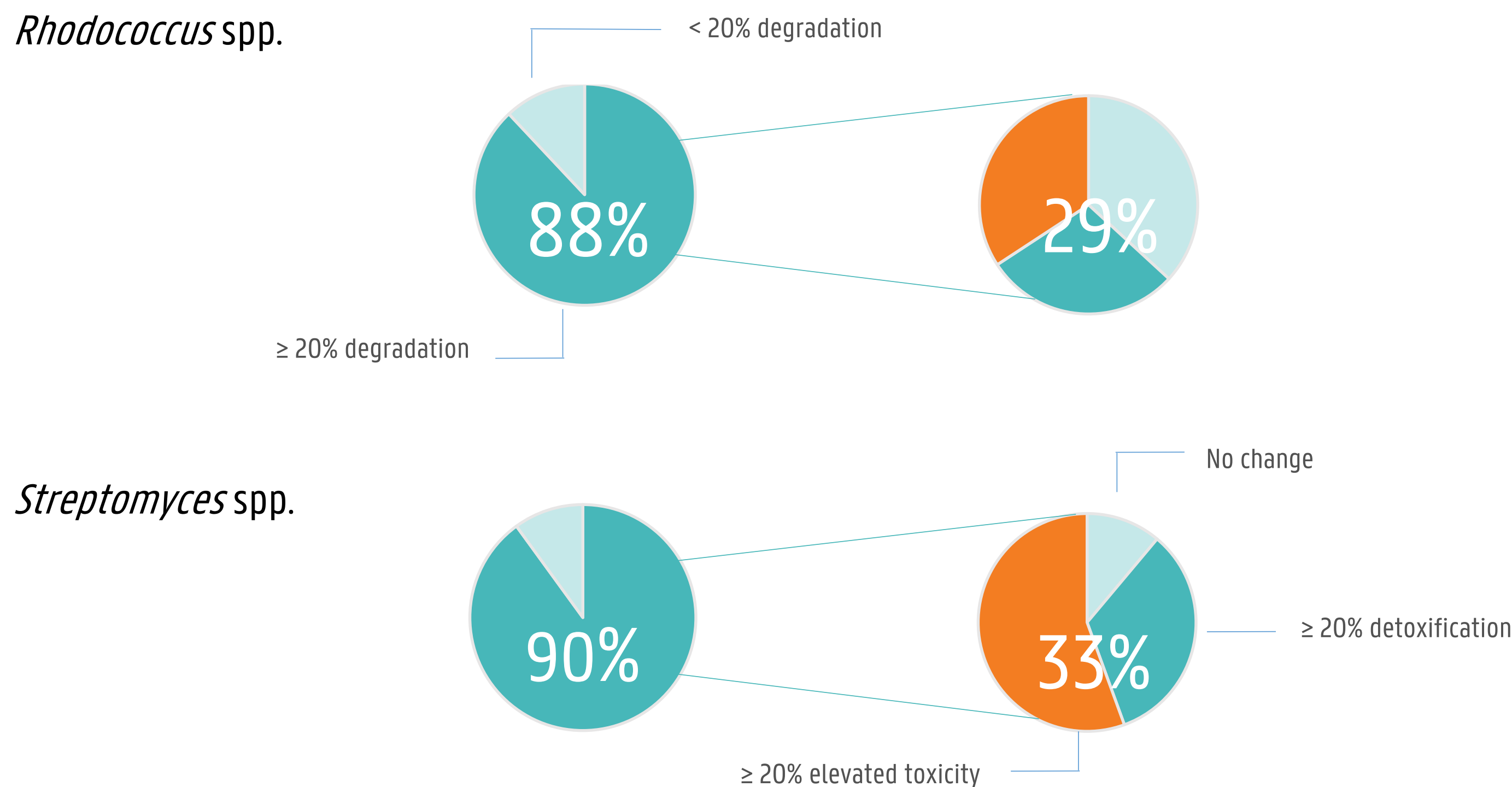

Overview of screening results. Forty-three Rhodococcus spp. and ten Streptomyces spp. were screened for the degradation and detoxification of ZEN. Eighty-eight and ninety percent respectively showed at least 20\% degradation, of which only 29 and 33\% was associated with at least $20 \%$ detoxification.

\section{Take home messages}

- Degradation of ZEN by Actinobacteria does not necessarily go hand in hand with detoxification. Also an increase in estrogenic toxicity, or no change in toxicity, have been observed.

- This highlights the importance of toxicity testing during the screening phase.

- For Rhodococcus sp. a high intra- and inter-species diversity in the degradation and detoxification of zearalenone is observed.
Higher estrogenic toxicity is not always related to known estrogenic metabolites

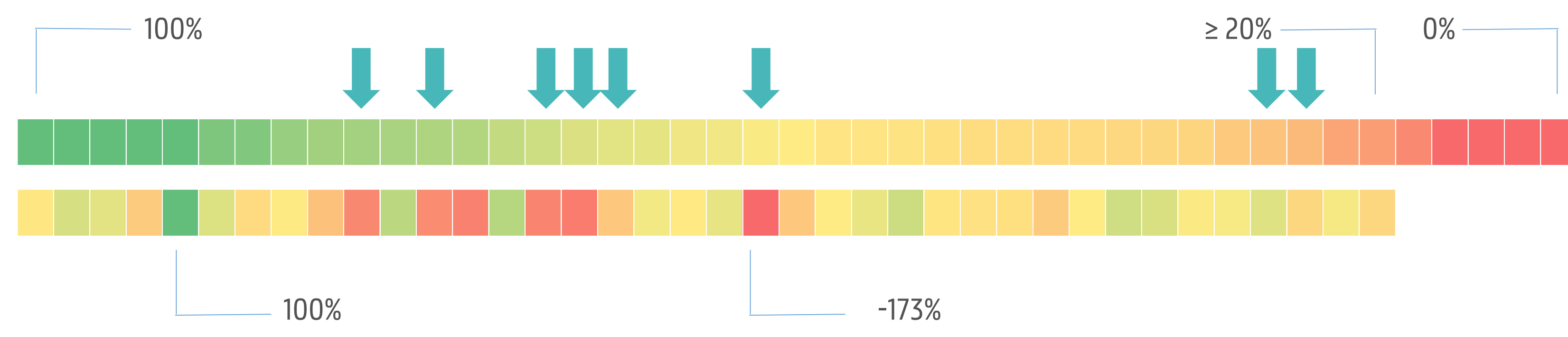

Specific results for the 43 screened Rhodococcus spp. The upper bar represents the strains arranged by Secreasing degradation percentage. The lower bar shows the detoxification (ranging from 100\% to -173\% or 73\% There

due arrows indicate samples where either only $\beta$-zearalenol or both $\alpha$ - and $\beta$-zearalenol were found.
High intraspecies diversity within

R. erythropolis

\begin{tabular}{l|c|c|c}
\hline & \multicolumn{1}{c}{ Degradation } & Detoxification \\
\hline R. erythropolis LMG 16262 & $100 \%$ & & $98 \%$ \\
\hline R. erythropolis BD2 & $92 \%$ & & $-16 \%$ \\
\hline R. erythropolis SQ1 & $85 \%$ & & $5 \%$ \\
\hline R. erythropolis LMG 16260 & $63 \%$ & & $13 \%$ \\
\hline R. erythropolis LMG 4059 & $25 \%$ & & $11 \%$, \\
\hline R. erythropolis R42661 & $0 \%$ & & $0 \%$ \\
\hline
\end{tabular}

Results for six different $R$. erythropolis strains. Both high degradation combined with lowered and increased toxicity were observed, as well as limited to absent degradation. 